\title{
No Laughing Matter: Intranasal Oxytocin Administration Changes Functional Brain Connectivity during Exposure to Infant Laughter
}

\author{
Madelon ME Riem ${ }^{1,2}$, Marinus H van IJzendoorn ${ }^{* 1,2}$, Mattie Tops ${ }^{1,2}$, Maarten AS Boksem ${ }^{3,4}$, \\ Serge ARB Rombouts ${ }^{2,5,6}$ and Marian J Bakermans-Kranenburg ${ }^{1,2}$ \\ 'Centre for Child and Family Studies, Leiden University, Leiden, The Netherlands; ' ${ }^{2}$ eeiden Institute for Brain and Cognition (LIBC), Leiden \\ University, Leiden, The Netherlands; ${ }^{3}$ Donders Institute for Brain, Cognition and Behavior, Radboud University, Nijmegen, The Netherlands; \\ ${ }^{4}$ Rotterdam School of Management, Erasmus University, Rotterdam, The Netherlands; ${ }^{5}$ Institute of Psychology, Leiden University, Leiden, \\ The Netherlands; ${ }^{6}$ Department of Radiology, Leiden University Medical Center, Leiden, The Netherlands
}

Infant laughter is a rewarding experience. It activates neural reward circuits and promotes parental proximity and care, thus facilitating parent-infant attachment. The neuropeptide oxytocin might enhance the incentive salience of infant laughter by modulating neural circuits related to the perception of infant cues. In a randomized controlled trial with functional magnetic resonance imaging we investigated the influence of intranasally administered oxytocin on functional brain connectivity in response to infant laughter. Blood oxygenation level-dependent responses to infant laughter were measured in 22 nulliparous women who were administered oxytocin and 20 nulliparous women who were administered a placebo. Elevated oxytocin levels reduced activation in the amygdala during infant laughter and enhanced functional connectivity between the amygdala and the orbitofrontal cortex, the anterior cingulate, the hippocampus, the precuneus, the supramarginal gyri, and the middle temporal gyrus. Increased functional connectivity between the amygdala and regions involved in emotion regulation may reduce negative emotional arousal while enhancing the incentive salience of the infant laughter.

Neuropsychopharmacology (2012) 37, 1257-1266; doi:10.1038/npp.201 I.313; published online 21 December 201 I

Keywords: oxytocin; infant laughter; amygdala; OFC; ACC; functional brain connectivity

\section{INTRODUCTION}

The laugh of an infant is a uniquely rewarding experience for parents. It provokes feelings of love and happiness and promotes infant survival by eliciting parental proximity and care (Bowlby, 1969/1982; Groh and Roisman, 2009; Mendes et al, 2009). Laughter is suggested to be the outcome of a long evolutionary history (van Hooff, 1972), and its production as well as perception might be hardwired in human beings (Owren and Bachorowski, 2003). Although infant smiling is one of the basic attachment behaviors that create closer proximity to a protective caregiver (Bowlby, 1969/1982; Sroufe and Waters, 1976) infant laughter is most easily released by tickling and other forms of rough-and-

\footnotetext{
*Correspondence: Professor Dr MH van IJzendoorn, Centre for Child and Family Studies, Leiden University, Wassenaarseweg 52, PO Box 9555, Leiden 2300 RB, The Netherlands, Tel: + 31715273434 ,

E-mail: vanijzen@fsw.leidenuniv.nl

Received 25 July 2011; revised 20 October 2011; accepted 9 November 2011
}

tumble play (Sroufe and Waters, 1976). The infant's laughter in its turn may activate neural reward centers in the parental brain (Kringelbach, 2005; Kringelbach et al, 2008; Strathearn et al, 2009) and reinforce parental playful interactions. Oxytocin is a neuropeptide that facilitates the onset of maternal behavior and mother-infant attachment (Carter, 1998; Galbally et al, 2011; Insel, 2010) and is involved in the perception of infant vocalizations (Riem et al, 2011a,b). Oxytocin might enhance the incentive salience of infant laughter by modulating neural circuits related to the perception of infant cues and thus motivating sensitive responsiveness to infant laughter. To our knowledge, this is the first randomized controlled study to examine the effects of intranasally administered oxytocin on functional brain connectivity in response to infant laughter.

Oxytocin administration studies have shown that it stimulates a range of social behaviors (for a meta-analysis, see Van IJzendoorn and Bakermans-Kranenburg, 2011) including empathy (Bartz et al, 2010a), mind-reading (Domes et al, 2007), trust (Kosfeld et al, 2005), in-group altruism (De Dreu et al, 2010), and paternal stimulating play 
(Naber et al, 2010). Feldman et al (2007) showed that maternal oxytocin levels across pregnancy are predictive of higher quality of postpartum maternal behavior. Oxytocin has anxiolytic and stress-reducing effects in breastfeeding mothers (Heinrichs et al, 2001; McCarthy et al, 1996) and this might increase mothers' sensitivity to infant signals including infant crying but also infant smiling and laughing. Taylor also suggested that oxytocin modulates stress responses and is implicated in the seeking of affiliative contact in response to stress (Taylor and Samson, 2005; Taylor, 2006). Although infant crying is very different from infant laughter, oxytocin seems to enhance sensitivity to both infant signals. For example, Strathearn et al (2009) found that mothers with a strong increase in peripheral oxytocin release while interacting with their infants show more activation in neural reward systems such as the OFC and the ventral striatum during the perception of their smiling infant than mothers with lower oxytocin levels (Strathearn et al, 2009). This finding might indicate that oxytocin increases the 'incentive salience' of infant laughter (Berridge, 2007).

One important target of oxytocin is the amygdala (MeyerLindenberg et al, 2011), a neural structure that is part of the neural network involved in emotional processing. In a previous study we found that oxytocin reduced amygdala responses to infant crying whereas it increased activation of the insula and inferior frontal gyrus (IFG) (Riem et al, 2011a), brain regions important for empathy, emotion understanding and maternal bonding (Bartels and Zeki, 2004; Shamay-Tsoory, 2011). The amygdala is also referred to as a neural hub because of its high degree of connectivity, which is critical for the flow and integration of information between regions (Pessoa, 2008). It is strongly connected with other brain regions involved in emotional processing such as the orbitofrontal cortex (OFC), the supra- and subgenual parts of the anterior cingulate cortex (ACC), the brainstem, and the thalamus (Bos et al, 2011; Pessoa, 2008).

Several studies have shown that by modulating amygdala activity hormones can shift neural output towards other brain regions within this network. For example, Kirsch et al (2005) showed that oxytocin reduces amygdala-brainstem coupling that is important for fear and arousal. Van Wingen et al (2010) showed that OFC-amygdala coupling was reduced after testosterone administration. The OFC is involved in reward and hedonic processing and it exhibits a specific neural response to infant stimuli (Kringelbach, 2005; Kringelbach et al, 2008; Noriuchi et al, 2008). Furthermore, previous studies have shown that the posterior OFC and ACC are involved in the perception of infant crying (Laurent and Ablow, 2011; Swain et al, 2008). Although most studies focused on infant crying only, Seifritz et al (2003) showed that the ACC was deactivated during both infant laughter and crying, indicating its important role in parent-infant interaction. Both structures are important for emotional regulation, in particular for the reduction of anxiety, by their inhibitory influence on the amygdala (Banks et al, 2007; Hahn et al, 2011; MeyerLindenberg et al, 2011; Stein et al, 2007; Swain et al, 2008). Thus, oxytocin might stimulate mother-infant bonding by modulating connectivity between the amygdala, ACC, and OFC and thereby enhancing the regulation of negative emotions and the experience of reward during motherinfant interaction (Bos et al, 2011).
In this study, we examined the influence of intranasally administered oxytocin on neural responses to infant laughter with functional magnetic resonance imaging (fMRI). Whole brain analysis was performed to explore the neural effects of oxytocin on functional activation during infant laughter. We focus our analyses on functional activation of the insula, IFG, and amygdala as a prior study showed that oxytocin modulated activation in these regions during the perception of infant crying (Riem et al, 2011a). Moreover, previous fMRI studies indicated that the insula and amygdala are involved in the perception of infant and adult laughter (Sander et al, 2003, 2007; Sander and Scheich, 2001, 2005; Seifritz et al, 2003). Furthermore, we examined oxytocin effects on the ventral striatum, the ACC, and the OFC because of the suggested significance of these regions in mother-infant bonding and reward processing (Berridge and Kringelbach, 2008; Kringelbach, 2005; Kringelbach et al, 2008; Seifritz et al, 2003; Strathearn et al, 2009). We expected that oxytocin administration would be related to increased activity in the ventral striatum, ACC, OFC, insula, and IFG, and decreased activity in the amygdala. In addition, with psychophysiological-interaction (PPI) analysis we examined whether oxytocin affects amygdalaconnectivity during the perception of infant laughter. In region of interest (ROI) analyses we tested whether oxytocin modulated functional connectivity between the amygdala, OFC, and ACC during infant laughter.

\section{MATERIALS AND METHODS}

\section{Participants}

Participants were selected from a larger study investigating caregiving responses and physiological reactivity to infant crying (Out et al, 2010). The original sample consisted of 50 male and 134 female adult twin pairs. Zygosity was determined on the basis of a zygosity questionnaire (Magnus et al, 1983) and additional genetic analysis of six polymorphisms; results indicated that 12 twin pairs $(6.5 \%$ of the sample) classified as monozygotic (MZ) on the basis of the questionnaire were in fact dizygotic (DZ). A group of 44 right-handed females were recruited, 22 from $\mathrm{MZ}$ twin pairs and 22 from DZ twin pairs, without children of their own, in good health, without hearing impairments and MRI contraindications, pregnancy, psychiatric or neurological disorders, and screened for alcohol and drug use. A between subject-design with twin siblings was used in order to avoid time effects (for example, decreased neural responses to infant laughing due to habituation) and to minimize preexisting differences (for example, age, child-rearing experiences, and genetics) between the oxytocin and placebo group. Two MZ siblings were excluded from the analyses because of excessive head movement during fMRI scanning (peak displacement $=4 \mathrm{~mm}$ ). Twin siblings of 10 participants did not participate because of MRI contraindications or other exclusion criteria, resulting in a sample of 30 participants from twin pairs ( $8 \mathrm{MZ}$, and $7 \mathrm{DZ})$ and 12 participants without twin sibling (4 MZ, and $8 \mathrm{DZ})$. The mean age of the participants was 28.71 years $(S D=6.93$, range, 22-49). The majority of the participants (71.4\%) used oral contraceptives. Permission for this study was obtained 
from the Medical Ethics Committee of the Leiden University Medical Center and all participants gave informed consent.

\section{Procedure}

Participants were invited preferably in the luteal phase of their (self-reported) menstrual cycle. During the luteal phase, plasma oxytocin levels are lower (Salonia et al, 2005) and more responsive to stimulation such as by nipple stimulation (Leake et al, 1984). Therefore, effects of oxytocin nasal administration might be more pronounced during the luteal phase. Approximately $40 \mathrm{~min}$ before the start of the fMRI data acquisition subjects took nasal spray containing oxytocin or placebo. Time between oxytocin/ placebo administration and data acquisition was similar to previous fMRI studies (Marsh et al, 2010; Riem et al, 2011a; Rimmele et al, 2009). Participants were instructed to comfortably position themselves on the scanner bed. Cushions were placed between the head coil and the participant in order to prevent head movement. Participants were instructed to attend to the sounds they would hear. Before drug administration and after fMRI scanning participants completed a mood questionnaire in order to track mood changes following drug administration. Participants rated on seven-point Likert scales how much anger, sadness, pleasantness, empathy, happiness, warmth, and calmness they felt. In addition, after fMRI scanning participants rated how healthy the infant laughter sounded, and how much warmth and affection they felt while listening to the laughing sounds. Furthermore, participants rated whether they felt irritated while listening to the control stimuli on five-point Likert scale (one $=$ not irritated, five $=$ irritated).

\section{Experimental Paradigm}

Participants listened to intensity-matched infant crying and infant laughter sounds with a duration of $6 \mathrm{~s}$ (samples 261 and 110 of the International Affective Digitized Sounds system, Bradley and Lang, 1999). These infant sounds have been used in a previous fMRI study (Seifritz et al, 2003). Neutral auditory control stimuli were created identical to the original auditory stimuli in terms of duration, intensity, spectral content, and amplitude envelope, but lacking recognizable qualities. The infant sounds and control sounds were presented in eight cycles, each cycle consisting of four sounds (cry, cry-control, laughter, laughter-control). The order of presentation of sounds within each cycle was random; the intertrial-interval was $6 \mathrm{~s}$. In this study we focus only on neural responses to infant laughter. Elsewhere we report on the functional activation during the perception of (a different set of) infant crying sounds (Riem et al, 2011a).

\section{Oxytocin vs Placebo}

One sibling from each twin pair was randomly assigned to the oxytocin condition and the other sibling to the placebo condition, resulting in a group of 22 participants who were administered oxytocin and a group of 20 participants who were administered a placebo. Participants without a twin sibling were also randomly assigned to the oxytocin and placebo condition. Because no twin pair was assigned to either the experimental or the placebo condition, data concerning the effects of the experimental administration of oxytocin were statistically independent. The use of twins randomized across conditions enhanced comparability of the groups in areas other than the experimental manipulation. Approximately $40 \mathrm{~min}$ before the start of the fMRI data acquisition subjects took six puffs of nasal spray containing $4 \mathrm{IU} /$ puff of oxytocin (24 IU total) or six puffs of a placebospray $(\mathrm{NaCl}$ solution) under supervision of the experimenter. Drug administration was double-blind. Menstrual phase and use of oral contraceptives were balanced across the placebo and oxytocin group: 11 participants in the oxytocin and 11 participants in the placebo group were in the luteal phase, whereas 8 participants in the oxytocin group and 9 participants in the placebo group were in the follicular phase. In all, 14 participants in the oxytocin group and 16 participants in the placebo group used oral contraceptives, whereas 8 participants in the oxytocin group and 4 participants in the placebo group did not use oral contraceptives.

\section{Image Acquisition}

Scanning was performed with a standard whole-head coil on a 3-T Philips Achieva MRI system (Philips Medical Systems, Best, the Netherlands) in the Leiden University Medical Center. First, a T1-weighted anatomical scan was acquired (flip angle $=8^{\circ}, 140$ slices, voxelsize $0.875 \times$ $0.875 \times 1.2 \mathrm{~mm}$ ). In addition, a high-resolution EPI scan was obtained (for registration purposes) ( $\mathrm{TR}=2.2 \mathrm{~s}$; $\mathrm{TE}=30 \mathrm{~ms}$, flip angle $=80^{\circ}, \quad 84$ slices, voxelsize $1.96 \times 1.96 \times 2.00 \mathrm{~mm})$. For fMRI, a total of $185 \mathrm{~T} 2 *$ weighted whole-brain EPIs were acquired $(\mathrm{TR}=2.2 \mathrm{~s}$; $\mathrm{TE}=30 \mathrm{~ms}$, flip angle $=80^{\circ}, 38$ transverse slices, voxelsize $2.75 \times 2.75 \times 2.75 \mathrm{~mm}(+10 \%$ interslice gap $))$. Participants listened to the sounds through MRI compatible headphones. In accordance with Leiden University Medical Center policy, all anatomical scans were examined by a radiologist from the Radiology department. No anomalous findings were reported.

\section{FMRI Data Analysis}

Data analysis was carried out using FSL version 5.98 (FMRIB's Software Library, www.FMRIb.ox.ac.uk/fsl, (Smith et al, 2004)). The following pre-statistics processing was applied: motion correction (Jenkinson et al, 2002), non-brain removal (Smith, 2002), spatial smoothing using a Gaussian kernel of full-width-at-half-maximum $8.0 \mathrm{~mm}$, and high-pass temporal filtering (highpass filter cutoff $=$ 100.0 s). Functional scans were registered to the highresolution EPI-images, which were registered to the T1-weighted images, which were registered to standard space (Jenkinson et al, 2002; Jenkinson and Smith, 2001).

In native space, functional activation was examined using general linear model analysis. Each sound (cry, cry-control, laughter, laughter-control) was modeled separately as a square-wave function. Each predictor was then convolved with a double gamma hemodynamic response function and its temporal derivative was added to the model, giving eight regressors. We assessed the contrast laugh $>$ laugh-control 
in order to identify regions involved in the perception of infant laughing.

Second, we examined psychophysiological interactions (PPI), that is, condition-dependent changes in the covariation of the response between a seed region and other brain regions (Friston et al, 1997). We used the left and right amygdala as seed regions because we were primarily interested in the modulation effects of oxytocin on functional connectivity with the amygdala. We extracted the mean time series for each participant from the left and the right amygdala, defined using the Harvard-Oxford subcortical atlas. These time series were then used as a physiological regressor in the model. We applied two separate models: one to analyze left amygdala connectivity, and one to study right amygdala connectivity. A contrast between laughter and laughter-control (laugh $>$ laugh-control) was created and used as psychological regressor. This regressor was convolved with a double gamma hemodynamic response function and its temporal derivative was added to the model. Furthermore, the cry sound and the cry-control sound were included in the model as two separate regressors, both convolved with a double gamma hemodynamic response function. The temporal derivatives of these two regressors were also added to the model. Finally, the interaction between the psychological regressor and the time series from the left or right amygdala was modeled, giving eight regressors. We assessed the positive and negative contrast of the interaction in order to examine condition-dependent changes in functional connectivity.

All first-level contrast images (laughter $>$ control and PPI) and the corresponding variance images were transformed to standard space and submitted to second-level mixed-effects group whole brain analyses. For functional activation and PPI analysis, group means were tested using one-sample $t$-tests and we tested for group differences using two-sample $t$-tests on these contrasts with the oxytocin $v s$ placebo group comparison (oxytocin $>$ placebo and oxytocin $<$ placebo). We included age, menstrual cycle (follicular or luteal phase), and use of oral contraceptives as confound regressors in the model in the analyses of the group means and group differences in the functional activation and PPI analysis. The statistical images were thresholded using clusters determined by $Z>2.3$ and a cluster corrected significance threshold of $p<0.05$ (Worsley, 2001).
In addition to the whole brain analyses, ROI analyses were performed in FSL to investigate changes in activation of $a$ priori specified regions. For the perception of infant vocalizations these regions are the amygdala, ventral striatum/nucleus accumbens, IFG, and the insula (Bos et al, 2011; Riem et al, 2011a). These were defined using the Harvard-Oxford (sub)cortical atlas (http://www.fmrib. ox.ac.uk/fsl/data/atlas-descriptions.html\#ho). ROI analyses were limited to these search regions, applying the same statistical threshold as for the whole brain analyses, but correcting only for the size of ROI volumes. For PPI, ROIs were the anterior cingulate and the OFC, again defined with the Harvard-Oxford cortical atlas. ROI analyses were only conducted when these regions were not significantly activated in the whole brain analysis. The selection of these ROIs was based on a neural model for the effects of neuropeptides on brain connectivity (Bos et al, 2011). The amygdala is strongly connected to the medial and posterior regions of the orbitofrontal and the rostral and caudal regions of the ACC, and it has been suggested that oxytocin shifts neural output towards these brain regions by modulating amygdala activity (Amaral and Price, 1984; Bos et al, 2011; Carmichael and Price, 1995; Kringelbach and Rolls, 2004). Mean $Z$-values for significantly activated regions were calculated using Featquery (http://www. fmrib.ox.ac.uk/fsl/feat5/featquery.html) for visualization purposes in figures.

\section{RESULTS}

To examine whether oxytocin affected neural responses to infant laughter we contrasted the oxytocin group with the placebo group (oxytocin laugh $>$ control $>$ placebo laugh $>$ control and oxytocin laugh $>$ control $<$ placebo ${ }^{\text {laugh }>\text { control }}$ ). There were no significant group differences in the whole brain analysis. ROI analysis showed that, compared with the placebo group, participants who received oxytocin showed reduced activation in the amygdala when they listened to infant laughter compared with control sounds. There was one significant cluster in the left amygdala and one significant cluster in the right amygdala (cluster 1 : size $=54$, peak $Z=2.79$, MNI coordinates $x, y, z(\mathrm{~mm})=-22,-10,-16$, cluster 2: size $=22$, peak $Z=2.87$, MNI coordinates $x, y, z$ $(\mathrm{mm})=24,-4,-24)$ (Figure 1). There were no significant effects of oxytocin in the other regions of interest.

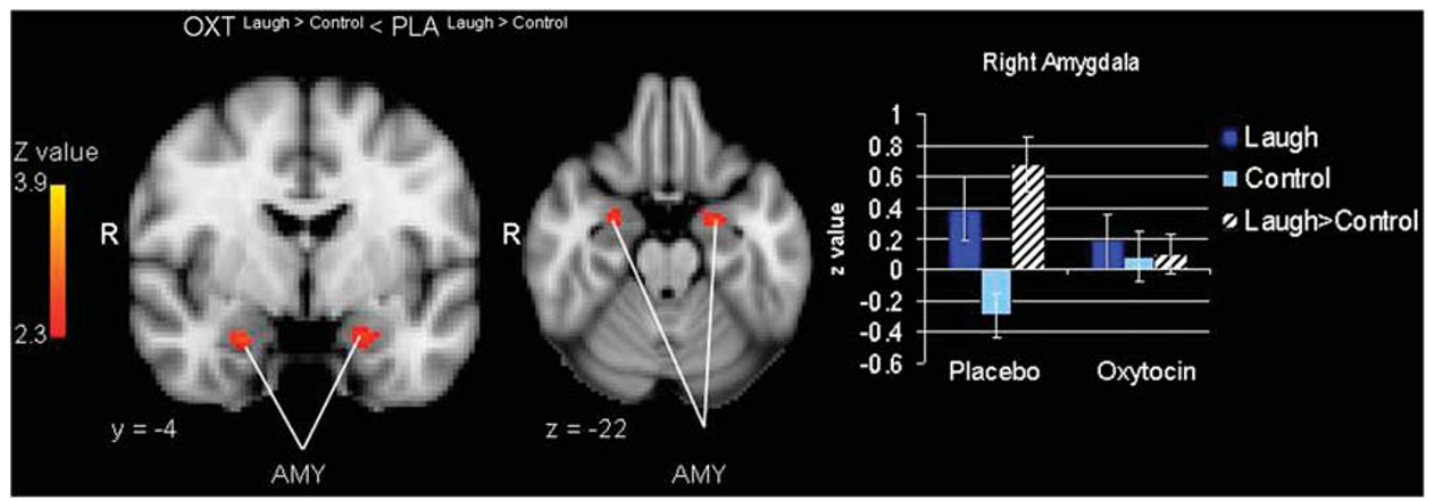

Figure I Oxytocin effect on bilateral amygdala (AMY) activation and mean Z-values and SEs of right amygdala activation during laugh, laugh-control, and laugh $>$ control in the oxytocin and placebo group. ROI analysis, $p<0.05$, corrected by cluster threshold $(Z>2.3)$. 
In the whole brain analysis of functional activation the contrast of infant laughter $v s$ control sound revealed two large clusters of activation in the placebo group with peak voxels in the superior temporal gyri (Cluster 1: size $=10831$ voxels, peak $Z=6.44$, MNI coordinates $x, y, z(\mathrm{~mm})=56$, $-14,-4$, Cluster 2: size $=7295$ voxels, peak $Z=6.30$, MNI coordinates $x, y, z(\mathrm{~mm})=-58,-12,0$, see Table 1 for an overview of statistics of the local maxima within these clusters). The pattern of activation included the bilateral temporal poles, the bilateral superior temporal gyrus, the bilateral OFC, the bilateral inferior and superior frontal gyrus, the bilateral amygdala, the brainstem, and the right putamen (see top panel Figure 2 for functional activation in the placebo group and lower panel Figure 2 for functional activation in the oxytocin group). ROI analyses indicated that there was no significant activation in the ventral striatum or in the insula during infant laughter compared with control sounds.

In the next step we performed PPI analyses to examine whether oxytocin affected functional connectivity with the amygdala when participants listened to infant laughter compared with control sounds. The whole brain analysis revealed that oxytocin significantly enhanced connectivity between the right amygdala and the left OFC, the bilateral hippocampus, the left precuneus, the right angular gyrus, and the right middle temporal gyrus during infant laughter compared with the control sound (see Table 2 and Figure 3 ). ROI analyses showed that functional connectivity between the bilateral amygdala and the caudal anterior cingulate was also enhanced by oxytocin during the exposure to infant laughter compared with control sounds (see Figure 3 and Table 2 for an overview of functional connectivity in the oxytocin and placebo group). The average laugh $>$ control

Table I MNI Coordinates and Z-max Values for Local Maxima Within the Significantly Activated Clusters During Exposure to Infant Laughter Compared with Control Sound in the Placebo Group

\begin{tabular}{llcrrr}
\hline Cluster & Region & $\boldsymbol{Z}$ & \multicolumn{3}{c}{ MNI coordinates } \\
\cline { 3 - 6 } & & & $\mathbf{x}$ & $\mathbf{y}$ & $\mathbf{z}$ \\
\hline 2 & R superior temporal gyrus & 6.44 & 56 & -14 & -4 \\
2 & R superior temporal gyrus & 5.45 & 66 & -24 & 6 \\
2 & R superior temporal gyrus & 5.42 & 50 & -4 & -18 \\
2 & R middle temporal gyrus & 4.92 & 50 & -12 & -16 \\
2 & R middle temporal gyrus & 4.55 & 62 & -46 & 12 \\
2 & R precentral gyrus & 4.41 & 60 & -2 & 46 \\
I & L superior temporal gyrus & 6.30 & -58 & -12 & 0 \\
I & L superior temporal gyrus & 6.12 & -62 & -24 & 4 \\
I & L planum temporale & 5.68 & -50 & -34 & 8 \\
I & L planum temporale & 5.49 & -48 & -32 & 4 \\
I & L superior temporal gyrus & 4.88 & -66 & -38 & 8 \\
I & L supramarginal gyrus & 4.32 & -64 & -42 & 18 \\
\hline
\end{tabular}

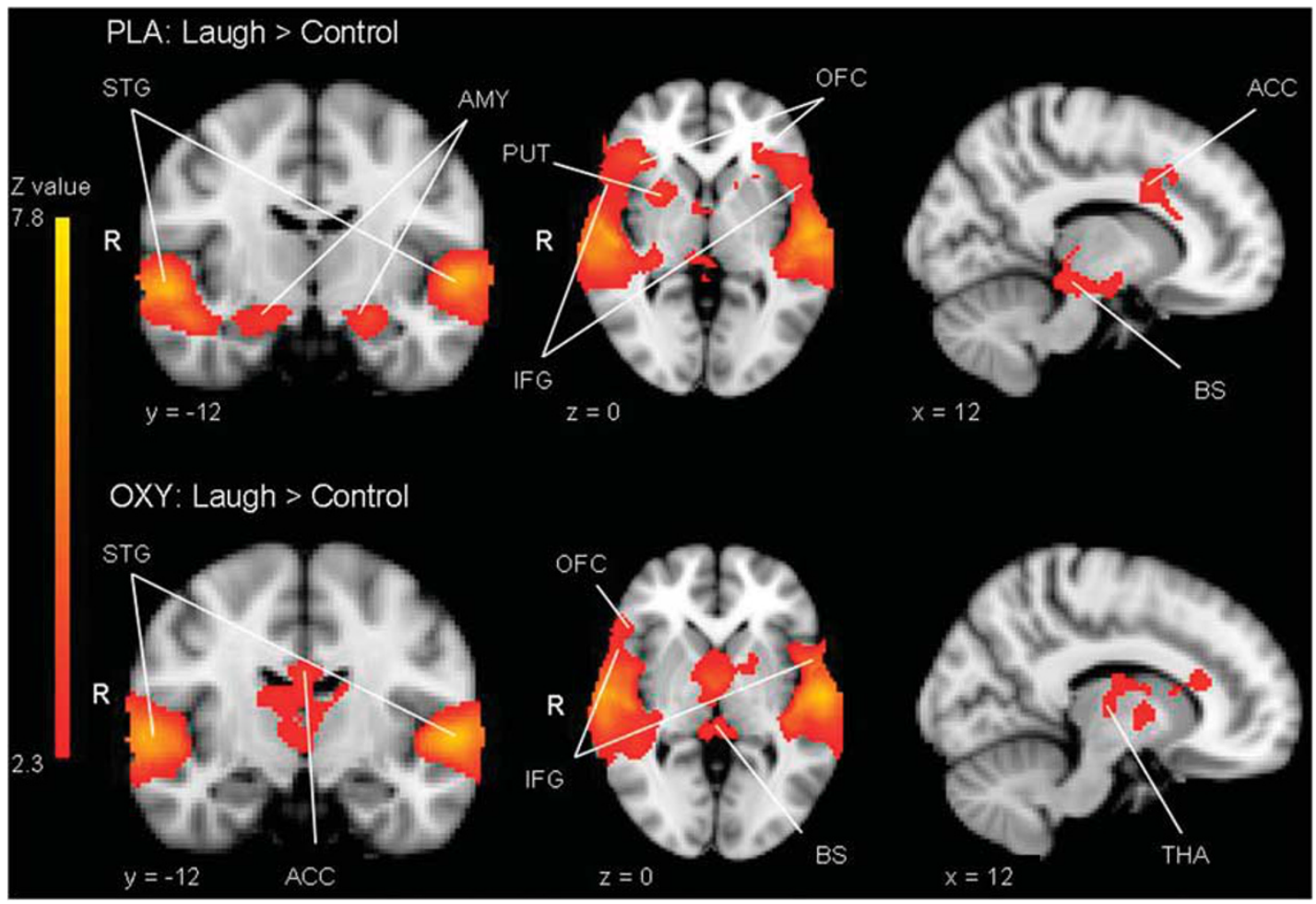

Figure 2 Top panel: significant activation in bilateral temporal poles, the bilateral superior temporal gyrus (STG), the bilateral OFC, the bilateral inferior and superior frontal gyrus (IFG), the bilateral amygdala (AMY), the brainstem (BS), the ACC, and the right putamen (PUT) for the contrast laugh > control in the placebo group. Lower panel: significant activation in bilateral temporal poles, the bilateral superior temporal gyrus, the right OFC, the bilateral IFG, the brainstem, the bilateral thalamus (THA), and the anterior cingulate for the contrast laugh $>$ control in the oxytocin group. Statistical images were thresholded with clusters determined by $Z>2.3$ and a cluster-corrected significance threshold of $p<0.05$. 
Table 2 Overview of Functional Connectivity During Infant Laughter Compared with Control Sound: MNI Coordinates, Cluster Size, and Z-max Values for Significant Clusters of Functional Connectivity

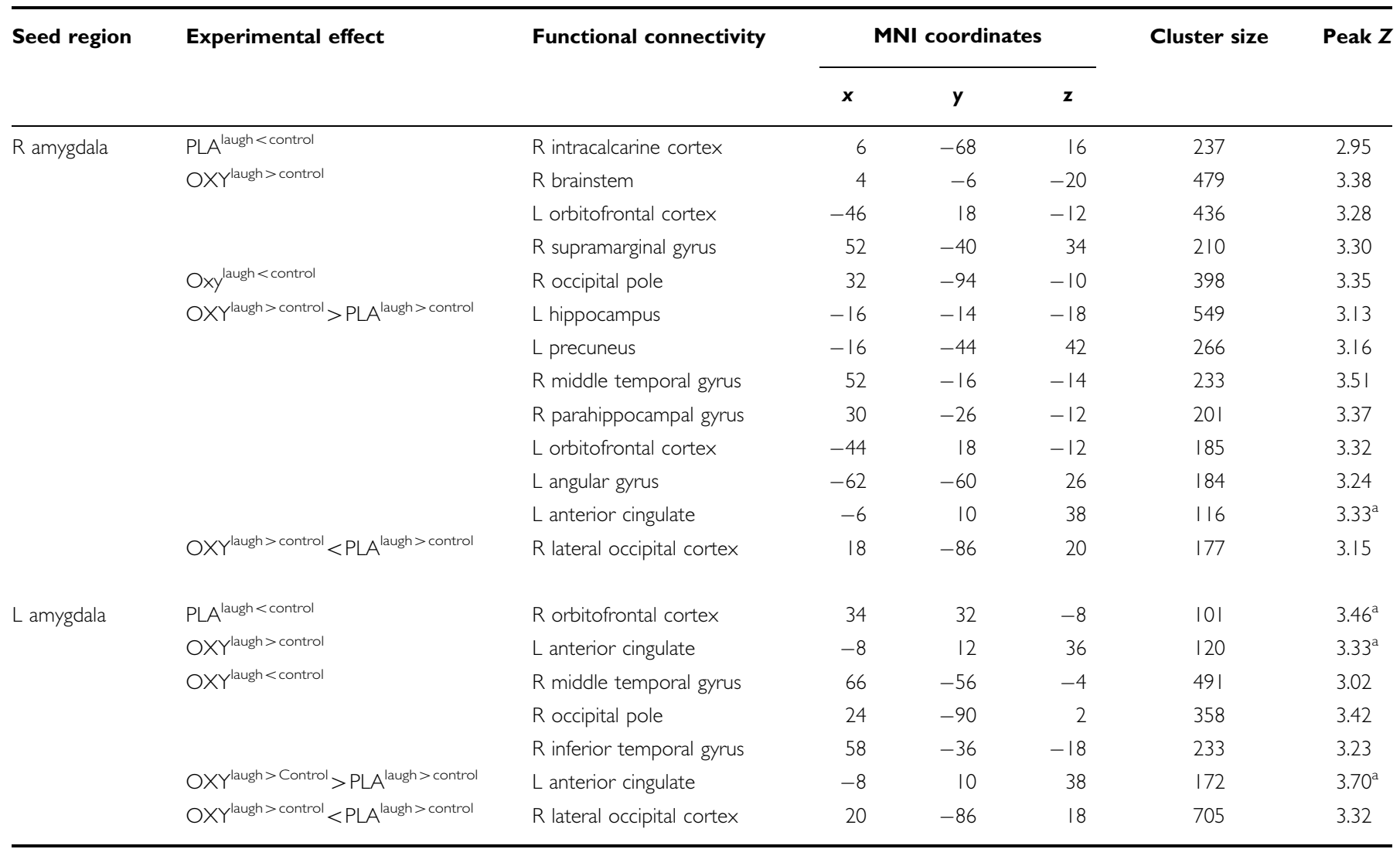

$p<0.05$, corrected by whole brain cluster threshold $(Z>2.3)$. Age, use of oral contraceptives, and menstrual cycle included as confound regressors in the model.

${ }^{a}$ Region of interest analysis, $p<0.05$, corrected by cluster threshold $(Z>2.3)$.

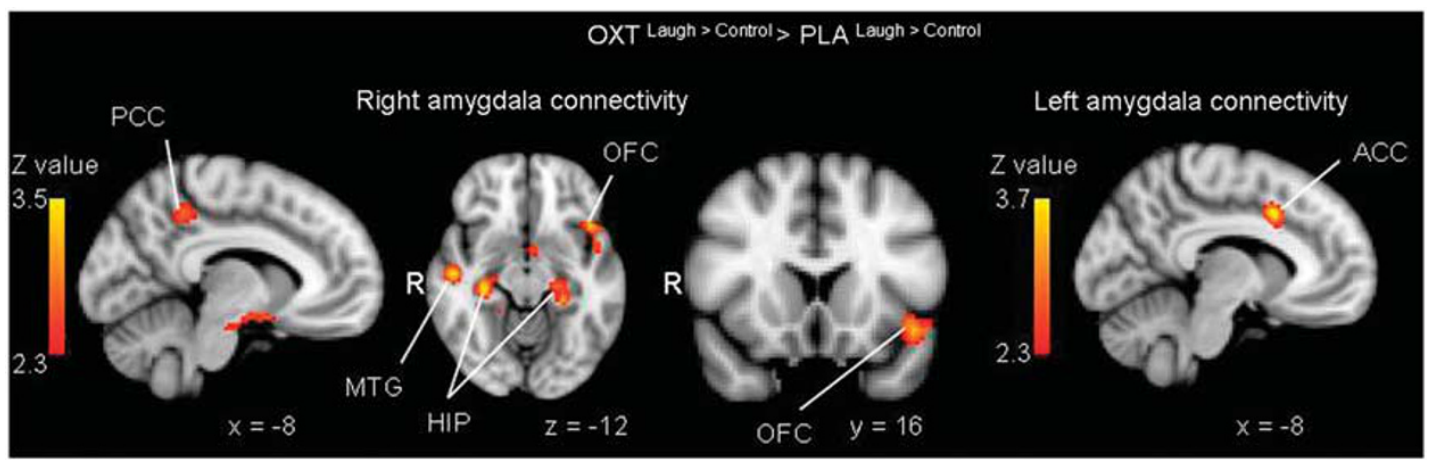

Figure 3 Left panel: oxytocin induced stronger functional connectivity between the right amygdala and the hippocampus (HIP), precuneus (PCC), OFC, and middle temporal gyrus (MTG) during the perception of infant laughter compared with control sound. Right panel: oxytocin also enhanced functional connectivity between the left amygdala and the ACC during the perception of infant laughter compared with control sound. Statistical images were thresholded with clusters determined by $Z>2.3$ and a cluster-corrected significance threshold of $p<0.05$.

connectivity change (or PPI) in the ACC in the oxytocin group was positive, but not significant (peak voxel oxytocin $>$ placebo comparison $x, y, z(\mathrm{~mm})=-6,10,38$, $Z=2.30$ ), whereas it is significantly increased as compared with the placebo PPI (peak voxel oxytocin $>$ placebo comparison $x, y, z(\mathrm{~mm})=-6,10,38, z=-2.56)$. Thus, there was a significant group difference although the PPI was not significant in the oxytocin group. We performed additional analyses examining whether left amygdala connectivity with the ACC was significantly different from right amygdala connectivity with the ACC. There was no significant difference $(\mathrm{F}(1,40)=0.19, p=0.89)$ and no significant hemisphere $\times$ treatment group (oxytocin $v s$ placebo) interaction $(\mathrm{F}(1,40)=1.07, p=0.31)$. In sum, oxytocin decreased amygdala activation relative to the placebo condition during exposure to infant laughter and 
increased functional coupling between the amygdala and regions implicated in the perception and regulation of emotional cues.

Independent-sample $t$-tests were used to examine the subjective rating of the laughing and control sounds. Participants who received oxytocin experienced more warm feelings $(\mathrm{M}=6.14, \mathrm{SD}=1.11)$ when listening to the laughing sounds compared with participants in the placebo group $(\mathrm{M}=5.25, \mathrm{SD}=1.62), t(39)=2.07, p<0.05$ (not significant after Bonferroni correction for multiple comparisons). However, there was no significant group difference in reported affection, $t(39)=1.14, p=0.26$ and no significant group difference in how healthy the participants rated the laughing sound $t(39)=0.49, p=0.63$. Participants did not feel much irritation while listening to the control sounds $(M=2.05, S D=1.32)$ and there was no significant group difference between the oxytocin and placebo group $t(39)=0.94, p=0.35$. To control for nonspecific effects of oxytocin on self-reported mood, we conducted repeatedmeasures analyses of variance with group (oxytocin and placebo) as between-subject factor and time (time 1: before drug administration, and time 2: after scanning) as withinsubject factor. There were no significant time $\times$ group interaction effects on any of the mood items: anger $F$ $(1,40)=0.07, p=0.80$, sadness $F(1,40)=0.22, p=0.64$, pleasantness $\mathrm{F}(1,40)=0.15, p=0.90$, empathy $\mathrm{F}(1,40)=$ $0.00, p=0.99$, happiness $\mathrm{F}(1,40)=2.69, p=0.11$, warm feeling $F(1,40)=0.36, p=0.55$, and calmness $F(1,40)=$ $0.01, p=0.91$.

\section{DISCUSSION}

Our study demonstrates that oxytocin reduces amygdala activation relative to the placebo condition when individuals listen to infant laughter compared with control sounds. Oxytocin has stress-reducing effects in lactating mothers (Heinrichs et al, 2001, 2003), which might enable them to be more sensitive to infant cues. Recent evidence indicates that inhibition of the amygdala, a brain region involved in anxiety and emotional arousal (LeDoux, 2000; Morrison and Salzman, 2010), might be the underlying neural mechanism of these calming oxytocin effects (Gamer et al, 2010; Kirsch et al, 2005; Riem et al, 2011a). In addition, we found that oxytocin increased functional connectivity between the amygdala and neural reward regions, the OFC and the caudal ACC (Berridge and Kringelbach, 2008; Haber and Knutson, 2010; Kringelbach, 2005). Our findings provide empirical support for the neural model on the effects of neuropeptides on brain connectivity proposed by Bos et al (2011). Increased functional connectivity between the OFC, ACC, and amygdala may promote mother-infant attachment by enhancing cognitive control over negative emotionality and at the same time increasing the incentive salience of infant laughter (Berridge, 2007; Berridge and Kringelbach, 2008; Bos et al, 2011).

Infant laughter is easily released during playful interactions with their parents, which are highly rewarding for both parent and infant (Feldman, 2003; Sroufe and Waters, 1976). Previous studies on oxytocin and parenting showed that oxytocin promotes such playful interactions. For example, Feldman et al (2010) showed that fathers with high levels of oxytocin displayed more stimulatory contact during play with their child. In a complementary study, Naber et al (2010) found that intranasally administered oxytocin enhances paternal playful interaction. The current study is the first to examine the neural mechanism underlying the effect of oxytocin on the perception of playful infant vocalizations. Future studies should examine the neural mechanisms underlying the rewarding effects of playful parent-infant interactions in a more real-life situation, for example, by studying neural activation and connectivity during the presentation of video fragments of parent-infant playful interactions.

Furthermore, we found that oxytocin increased functional connectivity between the amygdala and the hippocampus, middle temporal gyrus, and precuneus during infant laughter compared with control sound. Although in previous studies the hippocampus has not been directly implicated in parental care, it is known to be affected by parenting experiences, possibly by the altered parental hormonal levels after child birth (Leuner et al, 2010). Several studies indicate that amygdala-hippocampus interactions are crucial for emotional memory (Schaefer and Gray, 2007), an important factor in parenting that can be enhanced by oxytocin (Bartz et al, 2010b; Guastella et al, 2008; Rimmele et al, 2009). The middle temporal gyrus and precuneus are part of a network involved in the perception of speech and prosody (Leitman et al, 2010; Price, 2010; Turken and Dronkers, 2011) and in aspects of social cognition such as mentalizing and emotion understanding (Atique et al, 2011; Leitman et al, 2010). Pessoa (2008) suggested that the high degree of connectivity between the amygdala and other regions involved in emotional processing might serve the integration of emotion and cognition, and the evaluation of sensory information. Oxytocin might facilitate evaluation of and responding to emotional stimuli by modulating neural connectivity (Pessoa, 2008; Salzman and Fusi, 2010). This is supported by a study of Gamer et al (2010), who showed that oxytocin increased functional coupling between the amygdala and the superior colliculus as well as gaze changes towards the eyes of an emotional stranger in order to facilitate the classification of the emotion.

In the placebo group, we found an increase in activation in the temporal poles, the OFC, the inferior and superior frontal gyrus, the amygdala, the brainstem, and the putamen in response to infant laughter compared with control sounds. Previous fMRI studies on the perception of infant stimuli also reported activation in these regions. For example, Strathearn et al (2008) presented mothers with images of their own happy infants and found significant activation in the putamen, a subregion of the ventral striatum, which is important for reward processing. In a previous study on infant crying we also found significant activation in the IFG and the temporal poles (Riem et al, 2011a), regions that are involved in theory of mind and empathy (Chakrabarti et al, 2006; Decety and Jackson, 2004). However, in contrast with our previous study, the insula was not significantly activated during exposure to infant laughter and oxytocin did not modulate activation in this region. Previous studies have shown that the insula is involved in feeling empathy for others, in particular when 
observing others in pain (Lamm et al, 2011). For example, Lang et al (2011) indicated that the insula was significantly more activated when listening to pain expressions compared with positive stimuli such as laughter, which is in line with our respective results for activation during exposure to infant laughter and infant crying.

One limitation of this study is that the use of a betweensubjects design implies the risk of preexisting differences between the oxytocin and placebo group that might have influenced the results. However, most of our participants were $\mathrm{MZ}$ and $\mathrm{DZ}$ twin pairs, perfectly matched on age and global child-rearing experiences and even on genotype in MZ twin pairs. Second, it should be noted that neural responses to infant laughter might be affected by the infant crying sounds that were also presented during the experimental paradigm because the infant laughter-crying contrast might have enhanced the rewarding experience of infant laughter. In addition, the physiological effects induced by intranasally administered oxytocin are not well-understood and might be different from the effects of endogenous oxytocin secretion. Furthermore, functional connectivity using fMRI is a correlation method that does not allow conclusions about the (direction of the) causal relation between the OFC, ACC, and amygdala during exposure to infant laughter. Several studies suggest however that the OFC and ACC regulate negative emotionality by inhibiting the amygdala (Banks et al, 2007; Hahn et al, 2011; Stein et al, 2007; Swain et al, 2008). Furthermore, it should be noted that the regions in which we found significant connectivity changes in the oxytocin-placebo comparison did not all have a significant PPI in the oxytocin group. Finally, our findings can only be generalized to women without children. The perception of infant signals is influenced by parental status (Seifritz et al, 2003), possibly because of the altered oxytocinergic system after child birth, lactation, and parent-infant contact (Feldman et al, 2010). More research is needed to examine oxytocin effects on functional activation and connectivity during infant signals in parents. Intranasal oxytocin effects might be even more pronounced in parents, because of the emotional and biological significance of their own infant's laughing and crying.

Our findings support neural models on functional connectivity between the amygdala, OFC, and ACC proposed by Kringelbach (2005), Bos et al (2011), and MeyerLindenberg et al (2011). Kringelbach et al (2008) showed that the medial OFC exhibits a very early and specific pattern of activity to infant cues. Therefore, it has been speculated that the medial OFC might be the neural base for the innate releasing mechanism described by Lorenz (1943) for affection and care of young infants. The OFC might encode rewarding characteristics of infant stimuli such as 'cuteness' that predispose parents to perceive infant stimuli as special and that elicit nurturing. The medial and posterior OFC, ACC, and amygdala have strong reciprocal connections that are important for the effect of the encoded reward value on subsequent behavior, for example, caregiving responses (Kringelbach, 2005). Although the medial OFC and ACC have been suggested to be important hedonic hot spots of the brain (Berridge and Kringelbach, 2008; Kringelbach, 2005; Parsons et al, 2010), they are also involved in emotion regulation by their inhibitory influence on the amygdala, especially the supra- and subgenual parts of the ACC (Banks et al, 2007; Hahn et al, 2011; Stein et al, 2007). Bos et al (2011) suggested that oxytocin facilitates social bonding by enhancing cognitive control from prefrontal regions to regulate emotionality, as well as by its effects on the experience of reward during social interaction. Our findings are consistent with this model and suggest that oxytocin promotes parent-infant attachment by reducing negative emotional arousal while increasing the incentive salience of infant cues.

In conclusion, this is the first study to show the effects of oxytocin intranasal administration on functional activation and connectivity to infant laughter. We found that oxytocin decreases amygdala activation to infant laughter and increases functional connectivity between the amygdala and the OFC, ACC, and several other brain regions involved in emotional processing. Our results extend previous findings indicating a central role for oxytocin in parent-infant playful interactions and attachment formation (Feldman, 2003; Feldman et al, 2007; Naber et al, 2010). Increased functional connectivity between the amygdala, ACC, and OFC may stimulate mother-infant bonding by enhancing the regulation of negative emotions and the experience of reward during parent-infant interaction. Thus, oxytocin increases the incentive salience of infant laughter, which might be one of the mechanisms that lead to enhanced sensitive responsiveness to infant cues and playful parentinfant interaction.

\section{ACKNOWLEDGEMENTS}

We are grateful to Esther Valk and Dorothée Out for their contribution to data collection. We thank Thijs Schrama for his contribution to the control stimuli. Last but not least, we thank the twins who participated in the study. The authors SARBR, MT, MJBK, and MHvIJ were supported by awards from the Netherlands Organization for Scientific Research (NWO) (SARBR: VIDI Grant, MT: Veni Grant, MJBK: VIDI and VICI Grants; MHvIJ: SPINOZA prize).

\section{DISCLOSURE}

The authors declare no conflict of interest.

\section{REFERENCES}

Amaral DG, Price JL (1984). Amygdalo-cortical projections in the monkey (Macaca fascicularis). J Comp Neurol 230: 465-496.

Atique B, Erb M, Gharabaghi A, Grodd W, Anders S (2011). Taskspecific activity and connectivity within the mentalizing network during emotion and intention mentalizing. Neuroimage 55: 1899-1911.

Banks SJ, Eddy KT, Angstadt M, Nathan PJ, Phan KL (2007). Amygdala-frontal connectivity during emotion regulation. Soc Cogn Affect Neurosci 2: 303-312.

Bartels A, Zeki S (2004). The neural correlates of maternal and romantic love. Neuroimage 21: 1155-1166.

Bartz JA, Zaki J, Bolger N, Hollander E, Ludwig NN, Kolevzon A et al (2010a). Oxytocin selectively improves empathic accuracy. Psychol Sci 21: 1426-1428.

Bartz JA, Zaki J, Ochsner KN, Bolger N, Kolevzon A, Ludwig N et al (2010b). Effects of oxytocin on recollections of maternal care and closeness. Proc Natl Acad Sci USA 107: 21371-21375. 
Berridge KC (2007). The debate over dopamine's role in reward: the case for incentive salience. Psychopharmacology (Berl) 191: 391-431.

Berridge KC, Kringelbach ML (2008). Affective neuroscience of pleasure: reward in humans and animals. Psychopharmacology (Berl) 199: 457-480.

Bos PA, Panksepp J, Bluthé R-M, Honk JV (2011). Acute effects of steroid hormones and neuropeptides on human social-emotional behavior: a review of single administration studies. Front Neuroendocrinol, print copy in press (originally published on 21 January 2011, doi:10.1016/j.yfrne.2011.01.002).

Bowlby J (1969/1982). Attachment and Loss, vol 1. Basic Books: New York.

Bradley MM, Lang PJ (1999). International Affective Digitized Sounds (IADS): Stimuli, Instruction Manual and Affective Ratings. Technical Report B-2, The Center for Research in Psychophysiology, University of Florida: Gainsville, FL.

Carmichael ST, Price JL (1995). Limbic connections of the orbital and medial prefrontal cortex in macaque monkeys. J Comp Neurol 363: 615-641.

Carter CS (1998). Neuroendocrine perspectives on social attachment and love. Psychoneuroendocrinology 23: 779-818.

Chakrabarti B, Bullmore E, Baron-Cohen S (2006). Empathizing with basic emotions: common and discrete neural substrates. Soc Neurosci 1: 364-384.

De Dreu CK, Greer LL, Handgraaf MJ, Shalvi S, Van Kleef GA, Baas $M$ et al (2010). The neuropeptide oxytocin regulates parochial altruism in intergroup conflict among humans. Science 328: 1408-1411.

Decety J, Jackson PL (2004). The functional architecture of human empathy. Behav Cogn Neurosci Rev 3: 71-100.

Domes G, Heinrichs M, Michel A, Berger C, Herpertz SC (2007). Oxytocin improves 'mind-reading' in humans. Biol Psychiatry 61: 731-733.

Feldman R (2003). Infant-mother and infant-father synchrony: the coregulation of positive arousal. Infant Ment Health 24: 1-23.

Feldman R, Gordon I, Schneiderman I, Weisman O, ZagoorySharon O (2010). Natural variations in maternal and paternal care are associated with systematic changes in oxytocin following parent-infant contact. Psychoneuroendocrinology 35: $1133-1141$.

Feldman R, Weller A, Zagoory-Sharon O, Levine A (2007). Evidence for a neuroendocrinological foundation of human affiliation: plasma oxytocin levels across pregnancy and the postpartum period predict mother-infant bonding. Psychol Sci 18: $965-970$.

Friston KJ, Buechel C, Fink GR, Morris J, Rolls E, Dolan RJ (1997). Psychophysiological and modulatory interactions in neuroimaging. Neuroimage 6: 218-229.

Galbally M, Lewis AJ, IJzendoorn MH, Permezel M (2011). The role of oxytocin in mother-infant relations: a systematic review of human studies. Harv Rev Psychiatry 19: 1-14.

Gamer M, Zurowski B, Buchel C (2010). Different amygdala subregions mediate valence-related and attentional effects of oxytocin in humans. Proc Natl Acad Sci USA 107: 9400-9405.

Groh AM, Roisman GI (2009). Adults' autonomic and subjective emotional responses to infant vocalizations: the role of secure base script knowledge. Dev Psychol 45: 889-893.

Guastella AJ, Mitchell PB, Mathews F (2008). Oxytocin enhances the encoding of positive social memories in humans. Biol Psychiatry 64: 256-258.

Haber SN, Knutson B (2010). The reward circuit: linking primate anatomy and human imaging. Neuropsychopharm 35: 4-26.

Hahn A, Stein P, Windischberger C, Weissenbacher A, Spindelegger C, Moser E et al (2011). Reduced resting-state functional connectivity between amygdala and orbitofrontal cortex in social anxiety disorder. Neuroimage 56: 881-889.
Heinrichs M, Baumgartner T, Kirschbaum C, Ehlert U (2003). Social support and oxytocin interact to suppress cortisol and subjective responses to psychosocial stress. Biol Psychiatry 54: 1389-1398.

Heinrichs M, Meinlschmidt G, Neumann I, Wagner S, Kirschbaum C, Ehlert U et al (2001). Effects of suckling on hypothalamicpituitary-adrenal axis responses to psychosocial stress in postpartum lactating women. J Clin Endocrinol Metab 86: 4798-4804.

Insel TR (2010). The challenge of translation in social neuroscience: a review of oxytocin, vasopressin, and affiliative behavior. Neuron 65: 768-779.

Jenkinson M, Bannister P, Brady M, Smith S (2002). Improved optimization for the robust and accurate linear registration and motion correction of brain images. Neuroimage 17: 825-841.

Jenkinson M, Smith S (2001). A global optimisation method for robust affine registration of brain images. Med Image Anal 5: 143-156.

Kirsch P, Esslinger C, Chen Q, Mier D, Lis S, Siddhanti S et al (2005). Oxytocin modulates neural circuitry for social cognition and fear in humans. J Neurosci 25: 11489-11493.

Kosfeld M, Heinrichs M, Zak PJ, Fischbacher U, Fehr E (2005). Oxytocin increases trust in humans. Nature 435: 673-676.

Kringelbach ML (2005). The human orbitofrontal cortex: linking reward to hedonic experience. Nat Rev Neurosci 6: 691-702.

Kringelbach ML, Lehtonen A, Squire S, Harvey AG, Craske MG, Holliday IE et al (2008). A specific and rapid neural signature for parental instinct. PLoS One 3: e1664.

Kringelbach ML, Rolls ET (2004). The functional neuroanatomy of the human orbitofrontal cortex: evidence from neuroimaging and neuropsychology. Prog Beurobiol 72: 341-372.

Lamm C, Decety J, Singer T (2011). Meta-analytic evidence for common and distinct neural networks associated with directly experienced pain and empathy for pain. Neuroimage 54: 2492-2502.

Lang S, Yu T, Markl A, Müller F, Kotchoubey B (2011). Hearing others' pain: neural activity related to empathy. Cogn Affect Behav Neurosci 11: 386-395.

Laurent HK, Ablow JC (2011). A cry in the dark: depressed mothers show reduced neural activation to their own infants cry. Soc Cogn Affect Neurosci; e-pub ahead of print 5 January 2011, doi:10.1093/scan/nsq091.

Leake RD, Buster JE, Fisher DA (1984). The oxytocin secretory response to breast stimulation in women during the menstrual cycle. Am J Obstet Gynecol 148: 457-460.

LeDoux JE (2000). Emotion circuits in the brain. Annu Rev Neurosci 23: 155-184.

Leitman DI, Wolf DH, Ragland JD, Laukka P, Loughead J, Valdez JN et al (2010). 'It's not what you say, but how you say it': a reciprocal temporo-frontal network for affective prosody. Front Hum Neurosci 4: 19.

Leuner B, Glasper ER, Gould E (2010). Parenting and plasticity. Trends Neurosci 33: 465-473.

Lorenz K (1943). Die angeborenen Formen Moglicher Erfahrung. Zeitschrift fur Tierpsychologie 4: 235-519.

Magnus P, Berg K, Nance WE (1983). Predicting zygosity in Norwegian twin pairs born 1915-1960. Clin Genet 24: 103-112.

Marsh AA, Yu HH, Pine DS, Blair RJ (2010). Oxytocin improves specific recognition of positive facial expressions. Psychopharmacology (Berl) 209: 225-232.

McCarthy MM, McDonald CH, Brooks PJ, Goldman D (1996). An anxiolytic action of oxytocin is enhanced by estrogen in the mouse. Physiol Behav 60: 1209-1215.

Mendes D, Seidl-de-Moura ML, Siqueira JD (2009). The ontogenesis of smiling and its association with mothers' affective behaviors: a longitudinal study. Infant Behav Dev 32: 445-453.

Meyer-Lindenberg A, Domes G, Kirsch P, Heinrichs M (2011). Oxytocin and vasopressin in the human brain: social neuropeptides for translational medicine. Nat Rev Neurosci 12: 524-538. 
Morrison SE, Salzman CD (2010). Re-valuing the amygdala. Curr Opin Neurobiol 20: 221-230.

Naber F, Van IJzendoorn MH, Deschamps P, van Engeland H, Bakermans-Kranenburg MJ (2010). Intranasal oxytocin increases fathers' observed responsiveness during play with their children: a double-blind within-subject experiment. Psychoneuroendocrinology 35: 1583-1586.

Noriuchi M, Kikuchi Y, Senoo A (2008). The functional neuroanatomy of maternal love: mother's response to infant's attachment behaviors. Biol Psychiatry 63: 415-423.

Out D, Pieper S, Bakermans-Kranenburg MJ, van Ijzendoorn MH (2010). Physiological reactivity to infant crying: a behavioral genetic study. Genes Brain Behav 9: 868-876.

Owren MJ, Bachorowski JA (2003). Reconsidering the evolution of nonlinguistic communication: the case of laughter. J Nonverbal Behav 27: 183-200.

Parsons CE, Young KS, Murray L, Stein A, Kringelbach ML (2010). The functional neuroanatomy of the evolving parent-infant relationship. Prog Neurobiol 91: 220-241.

Pessoa L (2008). On the relationship between emotion and cognition. Nat Rev Neurosci 9: 148-158.

Price CJ (2010). The anatomy of language: a review of $100 \mathrm{fMRI}$ studies published in 2009. Ann NY Acad Sci 1191: 62-88.

Riem MME, Bakermans-Kranenburg MJ, Pieper S, Tops M, Boksem MAS, Vermeiren RRJM et al (2011a). Oxytocin modulates amygdala, insula, and inferior frontal gyrus responses to infant crying: a randomized controlled trial. Biol Psychiatry 70: 291-297.

Riem MME, Pieper S, Out D, Bakermans-Kranenburg MJ, Van IJzendoorn MH (2011b). Oxytocin receptor gene and depressive symptoms associated with physiological reactivity to infant crying. Soc Cogn Affect Neurosci 6: 294-300.

Rimmele U, Hediger K, Heinrichs M, Klaver P (2009). Oxytocin makes a face in memory familiar. J Neurosci 29: 38-42.

Salonia A, Nappi RE, Pontillo M, Daverio R, Smeraldi A, Briganti A et al (2005). Menstrual cycle-related changes in plasma oxytocin are relevant to normal sexual function in healthy women. Horm Beh 47: 164-169.

Salzman CD, Fusi S (2010). Emotion, cognition, and mental state representation in amygdala and prefrontal cortex. Annu Rev Neurosci 33: 173-202.

Sander K, Brechmann A, Scheich H (2003). Audition of laughing and crying leads to right amygdala activation in a low-noise fMRI setting. Brain Res Brain Res Protoc 11: 81-91.

Sander K, Frome Y, Scheich H (2007). FMRI activations of amygdala, cingulate cortex, and auditory cortex by infant laughing and crying. Hum Brain Mapp 28: 1007-1022.

Sander K, Scheich H (2001). Auditory perception of laughing and crying activates human amygdala regardless of attentional state. Brain Res Cogn Brain Res 12: 181-198.

Sander K, Scheich H (2005). Left auditory cortex and amygdala, but right insula dominance for human laughing and crying. J Cogn Neurosci 17: 1519-1531.
Schaefer A, Gray JR (2007). A role for the human amygdala in higher cognition. Rev Neurosci 18: 355-363.

Seifritz E, Esposito F, Neuhoff JG, Luthi A, Mustovic H, Dammann G et al (2003). Differential sex-independent amygdala response to infant crying and laughing in parents $v s$ nonparents. Biol Psychiatry 54: 1367-1375.

Shamay-Tsoory SG (2011). The neural bases for empathy. Neuroscientist 17: 18-24.

Smith SM (2002). Fast robust automated brain extraction. Hum Brain Mapp 17: 143-155.

Smith SM, Jenkinson M, Woolrich MW, Beckmann CF, Behrens TE, Johansen-Berg $\mathrm{H}$ et al (2004). Advances in functional and structural MR image analysis and implementation as FSL. Neuroimage 23(Suppl 1): S208-S219.

Sroufe LA, Waters E (1976). The ontogenesis of smiling and laughter: a perspective on the organization of development in infancy. Psychol Rev 83: 173-189.

Stein JL, Wiedholz LM, Bassett DS, Weinberger DR, Zink CF, Mattay VS et al (2007). A validated network of effective amygdala connectivity. Neuroimage 36: 736-745.

Strathearn L, Fonagy P, Amico J, Montague PR (2009). Adult attachment predicts maternal brain and oxytocin response to infant cues. Neuropsychopharm 34: 2655-2666.

Strathearn L, Li J, Fonagy P, Montague PR (2008). What's in a smile? Maternal brain responses to infant facial cues. Pediatrics 122: $40-51$.

Swain JE, Tasgin E, Mayes LC, Feldman R, Constable RT, Leckman JF (2008). Maternal brain response to own baby-cry is affected by cesarean section delivery. J Child Psychol Psychiatry 49: 1042-1052.

Taylor MM, Samson WK (2005). Stress hormone secretion is altered by central administration of intermedin/adrenomedullin-2. Brain Res 1045: 199-205.

Taylor SE (2006). Tend and befriend. Curr Dir Psychol Sci 15: 273-277.

Turken AU, Dronkers NF (2011). The neural architecture of the language comprehension network: converging evidence from lesion and connectivity analyses. Front Syst Neurosci 5: 1-20.

van Hooff J (1972). A comparative approach to the phylogeny of laughter and smiling. In: Hinde RA (ed). Non-verbal Communication. Cambridge University Press: Cambridge, England. pp 209-241.

Van IJzendoorn MH, Bakermans-Kranenburg MJ (2011). A sniff of trust: Meta-analysis of the effects of intranasal oxytocin administration on face recognition, trust to in-group, and trust to out-group. Psychoneuroendocrinology (in press; doi:10.1016/ j.psyneuen.2011.07.008).

Van Wingen G, Mattern C, Verkes RJ, Buitelaar J, Fernandez G (2010). Testosterone reduces amygdala-orbitofrontal cortex coupling. Psychoneuroendocrinology 35: 105-113.

Worsley KJ (2001). Statistical analysis of activation images. In: Jezzard PM MP, Smith SM (eds). Functional MRI: An Introduction to Methods. Oxford University Press: New York. pp 251-270. 